\title{
Real-Time EMG Signal Classification via Recurrent Neural Networks
}

\author{
Reza Bagherian Azhiri \\ Predictive Analytics and Technologies Lab, ME Dept. \\ The University of Texas at Dallas \\ Richardson, TX, USA \\ reza.azhiri@utdallas.edu
}

\author{
Mohammad Esmaeili \\ Department of Electrical and Computer Engineering \\ The University of Texas at Dallas \\ Richardson, TX, USA \\ esmaeili@utdallas.edu
}

\author{
Mehrdad Nourani \\ Predictive Analytics and Technologies Lab, ECE Dept. \\ The University of Texas at Dallas \\ Richardson, TX, USA \\ nourani@utdallas.edu
}

\begin{abstract}
Real-time classification of Electromyography signals is the most challenging part of controlling a prosthetic hand. Achieving a high classification accuracy of EMG signals in a short delay time is still challenging. Recurrent neural networks (RNNs) are artificial neural network architectures that are appropriate for sequential data such as EMG. In this paper, after extracting features from a hybrid time-frequency domain (discrete Wavelet transform), we utilize a set of recurrent neural network-based architectures to increase the classification accuracy and reduce the prediction delay time. The performances of these architectures are compared and in general outperform other state-of-the-art methods by achieving $96 \%$ classification accuracy in 600 msec.

Index Terms-Electromyography, Recurrent Neural Network, Real-Time classification, Deep Learning, Wavelet Transform.
\end{abstract}

\section{INTRODUCTION}

Electromyography (EMG)-based pattern recognition of finger movements has been widely accepted by researchers as a promising method for controlling of prosthetic hands. The EMG signal is the result of action potential of muscle tissues after contraction. This transmitted electrical signal is captured by skin-mounted sensors placed on the target muscle. Due to monotonic relation between finger movement and that of its associated EMG signal, the pattern recognition of hand gesture could be detected. This pattern recognition is examined in either offline or online mode. In offline mode, the major goal is to achieve a higher accuracy [1]. The extracted features from the EMG signal and utilized classifier both impact the accuracy. Generally speaking, three major feature sets are utilized for EMG signals classification: in time domain (TD), frequency domain (FD) and time-frequency domain. Various optimization methods such as Particle Swarm Optimization (PSO) and Genetic algorithm (GA) [2], [3] can be employed to select a set of features with significant importance. Also, a plethora number of classifiers have been reported by researchers including decision trees [4], random forest [5], $\mathrm{K}$ nearest neighbor $(\mathrm{KNN})$ [6], [7], naïve Bayes classifier [8], multilayer perceptron (MLP) [9], gradient boosting (GB) [10], support vector machine (SVM) [11], [12] and extreme value machine (EVM) [13].

Esa et al. [14] extracted Hudgins features, root mean square (RMS) and combination of all these features and then employed SVM as a classifier to get the accuracy of $86.67 \%$ on index finger and $96.67 \%$ on thumb finger. Reference [11] performed spectral analysis on EMG signals to extract reflection coefficients as the features and then implemented SVM as a classifier to get $89 \%$ accuracy. Azhiri et al. [15] has used the same features but implemented EVM and increased the accuracy to $91 \%$. Authors in [7] extracted fractional fast Fourier transform (FrFT) as their features, and then KNN was applied as a classifier to achieve the accuracy of $98.12 \%$. Bhattachargee et al. [10] extracted RMS, standard deviation, variance and FFT as their features and classified the results by GB classifier with $98.50 \%$ accuracy.

While the majority of studies have concentrated on the offline classification of EMG signals, the online performance of system has practical role on the real-time control of prosthetic hands. For such applications, not only the accuracy of a classifier but also the delay time to get that accuracy is important. Also, the accuracy of offline mode generally is more than online mode. Additionally, the accuracy in online systems depends on the delay time in the postprocessing. The less delay time is considered, the less accuracy is achievable.

Khushaba et al. [16] employed Hudgins features, and autoregressive (AR) for feature extraction and applied library SVM (LIBSVM) for classification. They got the average accuracy of $90 \%$ in $800 \mathrm{msec}$. In another online classification approach [17], wavelet packet transform as a generalized format of wavelet transform has been implemented on the EMG signals for feature extraction. Then by employing principal component analysis (PCA) and self-organizing feature map (SOFM), the number of features has been reduced. Finally, a multilayer perceptron (MLP) was used as a classifier. Jaramillo et al. [9] applied filtering and rectifying on original signals, extracted features in time, frequency and time-frequency 


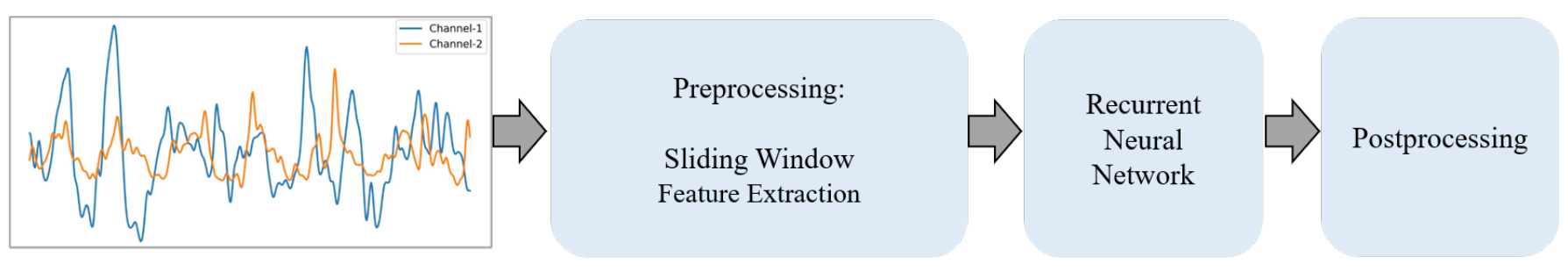

Fig. 1. The overall procedure of our proposed model.

domains, and utilized various parametric and nonparametric classifiers.

Recently, multifarious deep-learning based classifiers have been utilized in EMG-based hand gesture classification in online mode. Deep learning has the advantage of stability which reduces the errors associated with environmental noises especially in real-time EMG signal classification [18].

In classification with recurrent neural network (RNN), Nasri et al [19] achieved the accuracy of $77.85 \%$ on EMG dataset including 6 gestures with RNN structure. In order to enhance the accuracy of classification, Koch et el. [20] introduced a new loss function on the outputs of RNN in which true predictions have more weights. The accuracy for true predictions has improved by $10 \%$. Reference [21] compared different structures including feed-forward neural network (FFNN) as a static model and RNN, long-short term memory (LSTM) and gated recurrent units (GRU) as dynamic models to examine the temporal information of EMG signals. Using gesture detection accuracy as a criteria for the evaluation of proposed model, they concluded that both static and dynamic models have similar accuracies. Also, it seems using other techniques like ensemble learning [?] and other neural network architectures such as graph convolutional neural network (GCNN) [22], to make connection between different sensors, can significantly improve the classification accuracy.

In this paper, we investigate recurrent neural networkbased architectures for real-time classification of EMG signals. Discrete wavelet decomposition is employed to extract the features from time-frequency domain to feed into the recurrent neural networks. These architectures achieve a better classification accuracy in using shorter time in comparison with the state-of-the-art methods.

\section{Proposed Architecture}

Our proposed architecture, as shown in Fig. 1, includes three main steps: preprocessing, processing, and postprocessing.

\section{A. Preprocessing}

EMG signals have a stochastic behavior such that instantaneous processing is unable to generate favorite information for real-time classification of EMG signals. The input data is divided into the batches of consecutive samples called data windowing. Each window has the size of 400 samples with 200 overlaped samples with the previous window. Overlapped windows generally have better classification results than disjointed ones. The wavelet transform features are calculated for

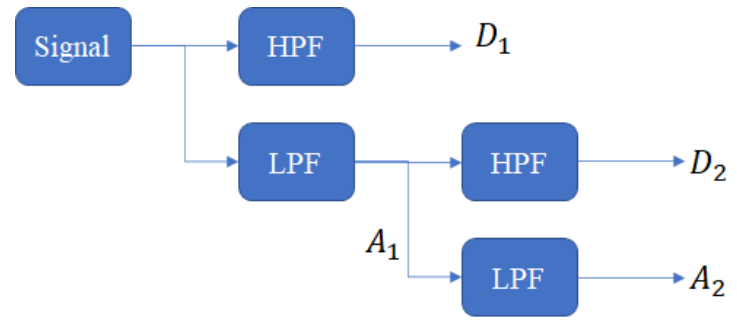

Fig. 2. Two-level wavelet Decomposition.
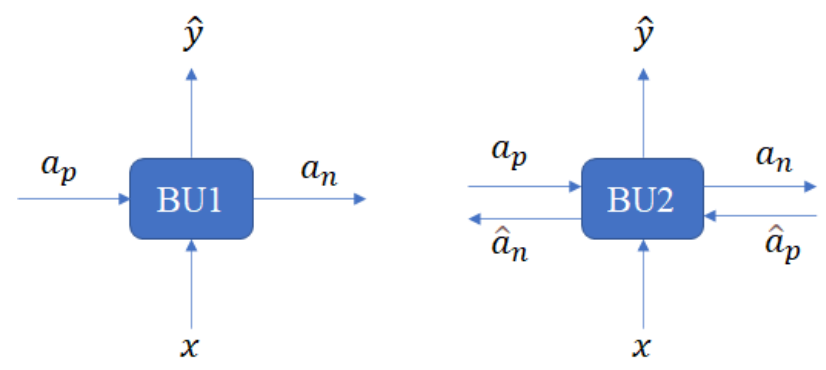

Fig. 3. Basic units BU1 and BU2.

each window and used as inputs to the RNN structure. In this paper, we consider a 2-level $d b l$ mother wavelet transform decomposition.

\section{B. Recurrent Neural Network Structures}

Recurrent neural networks (RNNs) are part of artificial neural networks that are appropriate to exhibit temporal dynamic behavior. RNNs are able to use their memory to process variable length sequences of inputs. This feature makes RNNs a valuable competitor against NNs and CNNs. RNNs have applications such as speech recognition and handwriting recognition, in dealing with sequence data.

Now we introduce two basic units (BUs) that are employed in our architectures. Fig. 3 illustrates two basic units BU1 and BU2.

- BU1: consider $W_{i}, b_{i}$ and $w_{a}$ as the parameters of the basic unit 1. This basic unit takes vectors $x$ and $a_{p}$ as its inputs and returns two outputs including $\hat{y}=A_{3}$ and 


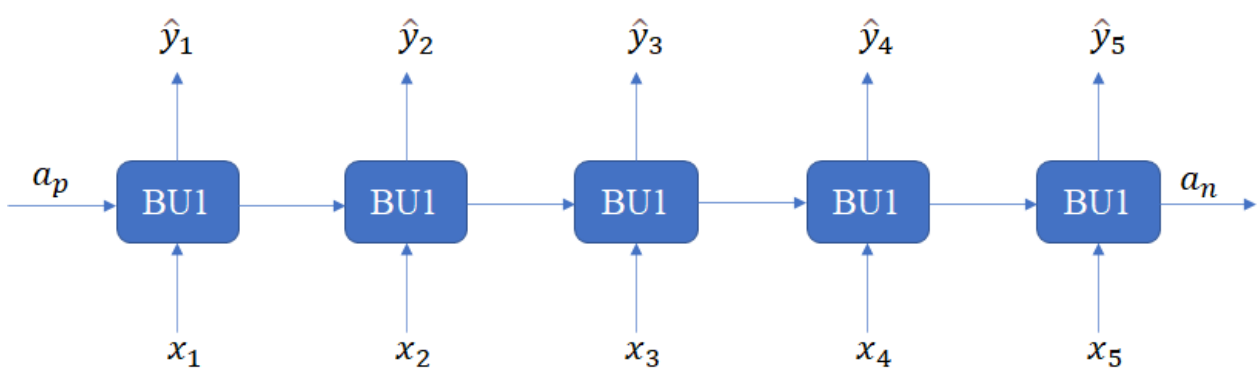

Fig. 4. The recurrent neural network architecture used in this paper.

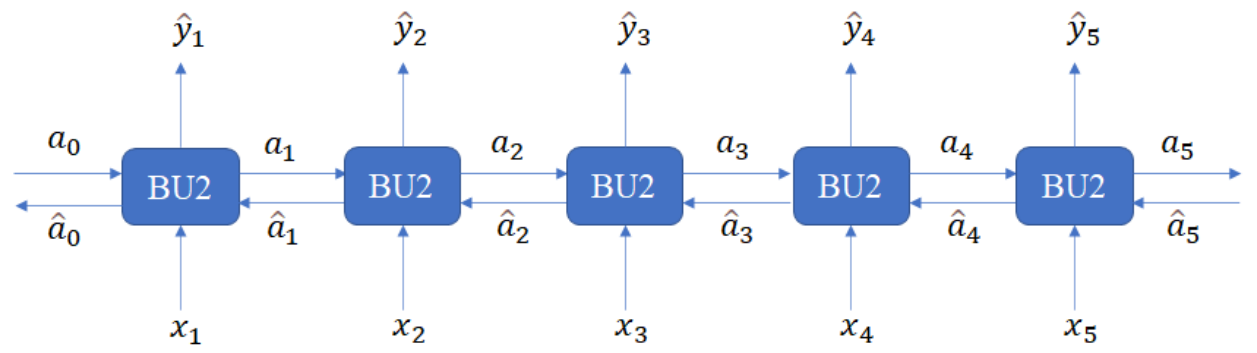

Fig. 5. The bidirectional recurrent neural network (BRNN) architecture used in this paper.

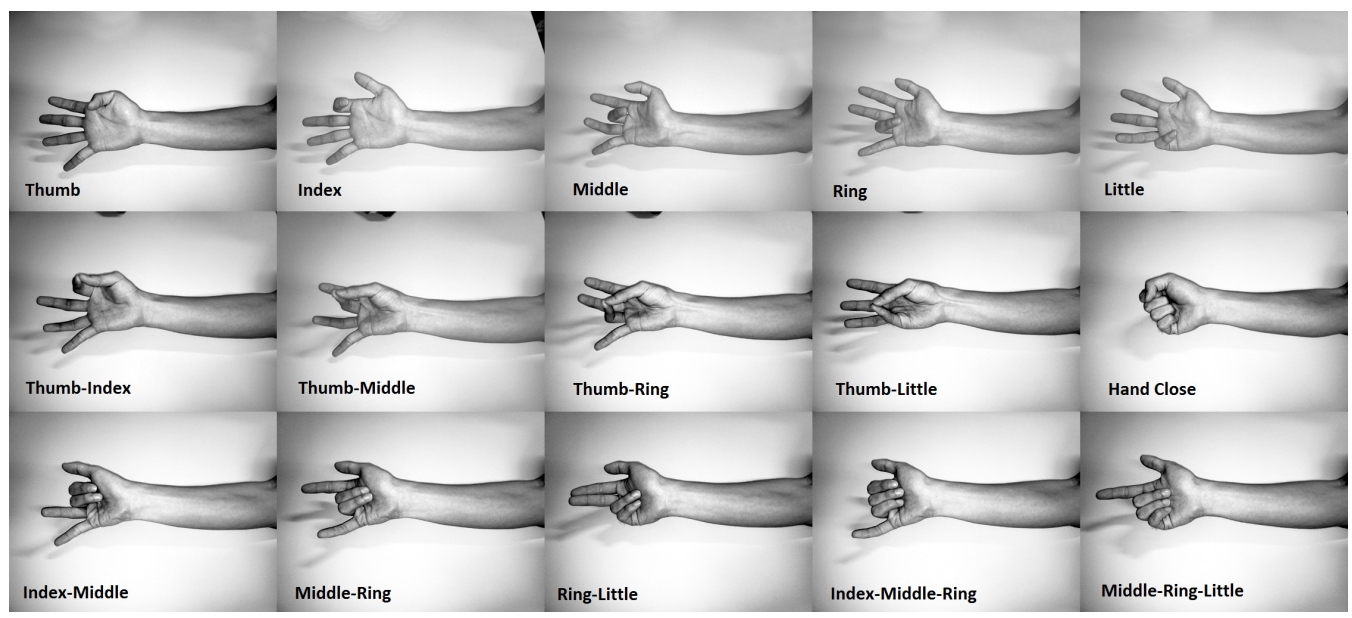

Fig. 6. Fifteen finger movements classes for individual and combined fingers [23] Each finger movement has related to a class as: (1. Thumb, 2. Index, 3. Middle, 4. Ring, 5. Little, 6. Thumb-Index, 7. Thumb-Middle, 8. Thumb-Ring, 9. Thumb-Little, 10. Index-Middle (I-M), 11. Middle-Ring (M-R), 12. Ring-Little (RL), 13. Index-Middle-Ring (I-M-R), 14. Middle-Ring-Little (M-RL), and 15. close hands (HC). )

$a_{n}=Z_{3}$ where

$$
\begin{aligned}
& A_{0}=x \\
& Z_{i+1}=W_{i} A_{i}+b_{i} \text { for } i=0,1,2 \\
& A_{i+1}=\tanh \left(Z_{i+1}+w_{a} a_{p}\right) \text { for } i=0 \\
& A_{i+1}=\tanh \left(Z_{i+1}\right) \text { for } i=1 \\
& A_{i+1}=\operatorname{Softmax}\left(Z_{i+1}\right) \text { for } i=2 .
\end{aligned}
$$

- BU2: consider $W_{i}, b_{i}, w_{a}$ and $w_{\hat{a}}$ as the parameters of the basic unit 1 . This basic unit takes vectors $x, a_{p}$ and $\hat{p}_{p}$ as its inputs and returns three outputs including $\hat{y}=A_{3}$ and $a_{n}=a_{p}=Z_{3}$ where

$$
A_{i+1}=\tanh \left(Z_{i+1}+w_{a} a_{p}+w_{\hat{a}} \hat{a}_{p}\right) \text { for } \quad i=0 .
$$

We construct an original recurrent neural network (RNN) and a bidirectional recurrent neural network (BRNN) by stacking a set of the basic units BU1 and BU2, respectively. The number of basic units that are used at each architecture is a hyperparameter which is tuned empirically. In this paper, five basic units are employed for each architecture. Also, we consider two different input approaches for each architecture as follows:

- Same inputs: in this approach, all the basic unit inputs 
are the same, i.e.,

$$
x_{1}=x_{2}=x_{3}=x_{4}=x_{5}=f\left(w_{i}\right)
$$

where $f\left(w_{i}\right)$ refers to the extracted features of window $w_{i}$ from the original signal. In this method, since all the basic unit inputs are the same, the RNN structure tries to improve the uncertainty at each basic unit during the training phase.

- Sequential inputs: in this approach, the basic unit inputs are different. Indeed, the input of each basic unit is the extracted features from a shifted window $(50 \mathrm{msec})$ of the original signal, i.e.,

$$
x_{j}=f\left(w_{i+j-1}\right) \quad \text { for } \quad j=1, \cdots, 5 .
$$

In this method, since the basic unit inputs are different, the RNN structure tries to learn the relationship between the inputs to reduce the uncertainty during the training phase [24].

\section{Postprocessing}

To refine the results of RNN structure, a postprocessing technique after classification of RNN structure is necessary. Each window performs a class decision, and postprocessing step minimizes ambiguities and false misclassifications [16]. In fact, postprocessing step helps us to combine the results gained by each window of the EMG signal. In this paper, the majority voting approach is selected in which the elements of each class is counted and a class as identified by majority is chosen.

\section{FEATURE EXTRACTION}

In the time domain, feature extraction functions are directly applied on a window of raw signals. Feature extraction functions are able to extract statistical features from the raw signal. There are several features that are commonly used in the literature. In this paper, we will use 19 of these features and their corresponding functions. Table II summarizes the mathematical expressions of these functions.

Discrete wavelet decomposition (DWD) provides sufficient information both for analysis and synthesis of the original signal, with a significant reduction in the computation time. Discrete wavelet transform can be implemented with a single level or multiple levels. A two-level discrete wavelet decomposition is shown in Fig. 2 .

The discrete wavelet transform of a signal is calculated by passing it through a series of filters. The samples are passed through a low pass filter. The signal is also decomposed simultaneously using a high-pass filter. The outputs give the detail coefficients (from the high-pass filter) and approximation coefficients (from the low-pass filter). It is important to note that these two filters are related to each other and they are known as a quadrature mirror filter. The decomposition is repeated to further increase the frequency resolution and the approximation coefficients decomposed with the high-pass and the low-pass filters and then down-sampled. After passing a window of raw signal through the high-pass and low-pass filters, the feature extraction functions in Table $\mathrm{I}$ are applied on the detail coefficients and approximation coefficients. In this paper, we consider a 2-level $d b l$ mother wavelet transform decomposition. Therefore, for a two-level wavelet decomposition, we will extract 57 features (3 layers of 19 wavelet features) for each window of the raw signal.

\section{EXPERIMENTAL RESULTS}

\section{A. Dataset}

The datasets which are used in this paper are provided by Center of Intelligent Mechatronic Systems at the University of Technology at Sydney [16]. The datasets includes EMG data of eight participants (six men and two women in range of 20 to 30 years old). EMG signals are sensitive to the condition of experiment and effects of neighbor limbs. For uniformity, participants sat on the armchair such that their arms were fixed and stable. To firmly stick the sensors to the skin above the targeted muscle, an adhesive skin interface was used. Resulting EMG signals from the electrodes were amplified to get the total gain of 1000 . The data was recorded at 4,000 $\mathrm{Hz}$ applying an analog-to-digital converter, a bandpass filter between 20 and $40 \mathrm{~Hz}$ and a notch filter to remove the $50 \mathrm{~Hz}$ line interference. This filtering is necessary to remove noises resulting by motion of artifacts and high-frequency random noise. Participants performed each movement of fingers six times while the duration of each movement from rest pose to contraction pose is five seconds.

In the first dataset, participants performed ten different finger movements consisting five individuals shown as thumb (T), index (I), middle (M), ring (R), little (L) and five combined movements named as thumb-index (T-I), thumb-middle (T-M), thumb-ring(T-R), thumb-little (T-L) and closed hands using two-channel (2C) sensors. We select the first four trials for the training of the classification methods and the last two for the test of the methods in order to check the accuracy of the classifiers. Each gesture took five seconds including rest and holding of each finger posture.

Second dataset includes total of 15 classes: five individuals finger movement thumb (T), index (I), middle (M), ring (R), little (L) and ten combined finger movements including thumbindex (T-I), thumb-middle (T-M), thumb-ring (T-R), thumblittle (TL), index-middle (I-M), middle-ring (M-R), ring-little (RL), index-middle-ring (I-M-R), middle-ring-little (M-RL), and close hands (HC) using eight-channel (8C) sensors. Each gesture took 20 seconds including rest and holding of each finger gesture. The first two trials were selected for the training of the model and the last one was used for the test to check the accuracy of proposed classifiers. Fig. 6 depicts these datasets that first ten finger movements are considered in the first dataset and entire fifteen movements are considered in the second dataset. The details of dataset, number of subjects and condition of experiments are reported in Table II.

\section{B. Results and Discussion}

In this section, the classification results of RNN and bidirectional RNN (BRNN) are presented and compared. Table III 
TABLE I

FEATURE EXTRACTION FUNCTIONS USED IN THIS PAPER,

\begin{tabular}{|c|c|c|}
\hline Statistical Metric & Abbreviation & Formula \\
\hline Integrated EMG 25 & IEMG & $\sum_{n=1}^{N}|x[n]|$ \\
\hline Mean Absolute Value [26] & MAV & $\frac{1}{N} \sum_{n=1}^{N}|x[n]|$ \\
\hline Simple Square Integrated [27] & SSI & $\sum_{n=1}^{N} x[n]^{2}$ \\
\hline Root Mean Square |27| & RMS & $\sqrt{\frac{1}{N} \sum_{n=1}^{N} x[n]^{2}}$ \\
\hline Variance 28 & VAR & $\frac{1}{N-1} \sum_{n=1}^{N} x[n]^{2}$ \\
\hline Myopulse Percentage Rate [27] & MYOP & $\frac{1}{N} \sum_{n=1}^{N} f(|x[n]|), \quad f(a)=\left\{\begin{array}{cc}1 & \text { if } a>T \\
0 & \text { otherwise }\end{array}\right.$ \\
\hline Waveform Length 27$]$ & WL & $\sum_{n=1}^{N-1}|x[n+1]-x[n]|$ \\
\hline Difference Absolute Mean Value $\mid 28$ & DAMV & $\frac{1}{N-1} \sum_{n=1}^{N-1}|x[n+1]-x[n]|$ \\
\hline Second-Order Moment 28 & M2 & $\sum_{n=1}^{N-1}(x[n+1]-x[n])^{2}$ \\
\hline Difference Variance Version $|28|$ & DVARV & $\frac{1}{N-2} \sum_{n=1}^{N-1}(x[n+1]-x[n])^{2}$ \\
\hline Difference absolute standard deviation value 28 & DASDV & $\sqrt{\frac{1}{N-1} \sum_{n=1}^{N-1}(x[n+1]-x[n])^{2}}$ \\
\hline Maximum [29] & MAX & $\max x[n]$ \\
\hline Minimum 29] & MIN & $\min x[n]$ \\
\hline Willison Amplitude [27] & WAMP & $\sum_{n=1}^{N-1} f(|x[n+1]-x[n]|), \quad f(a)=\left\{\begin{array}{cc}1 & \text { if } a>T \\
0 & \text { otherwise }\end{array}\right.$ \\
\hline Integrated Absolute of Second Derivative [13] & IASD & $\sum_{n=1}^{N-2}\left|x^{\prime}[n+1]-x^{\prime}[n]\right|, \quad x^{\prime}[n]=x[n+1]-x[n]$ \\
\hline Integrated Absolute of Third Derivative [13] & IATD & $\sum_{n=1}^{N-3}\left|x^{\prime \prime}[n+1]-x^{\prime \prime}[n]\right|, \quad x^{\prime \prime}[n]=x^{\prime}[n+1]-x^{\prime}[n]$ \\
\hline Integrated Exponential of Absolute Values $[13$ & IEAV & $\sum_{n=1}^{N} \exp (|x[n]|)$ \\
\hline Integrated Absolute Log Values 13 & IALV & $\sum_{n=1}^{N}|\log (x[n]+T)|$ \\
\hline Integrated Exponential [13] & IE & $\sum_{n=1}^{N} \exp (x[n])$ \\
\hline
\end{tabular}

TABLE II

SPECIFICATIONS OF EMG DATASETS.

\begin{tabular}{lll}
\hline Specifications & 2C Dataset & 8C Dataset \\
\hline \hline Number of electrodes & 2 & 8 \\
Number of finger movements & 10 & 15 \\
Number of repititions & 3 & 3 \\
Total number of repitions per subject & 30 & 45 \\
Number of subjects & 8 & 8 \\
Total number of repititions & 360 & 360 \\
Time for each repitition & $5 \mathrm{sec}$ & $20 \mathrm{sec}$ \\
Sampling rate & $4000 \mathrm{~Hz}$ & $4000 \mathrm{~Hz}$ \\
Resolutions & $12 \mathrm{bit}$ & $12 \mathrm{bit}$ \\
Window length/overlap & $100 / 50 \mathrm{msec}$ & $100 / 50 \mathrm{msec}$ \\
\hline
\end{tabular}

compares the test accuracy of proposed RNN architectures at different signal lengths for the first dataset. The method which is used for the postprocessing is majority voting. The results show that BRNN with the same inputs could achieve the higher accuracy in $600 \mathrm{msec}$ which is the best performance among all other proposed architectures of RNN. Due to the two-sided connectivity of basic units, the BRNN architecture is able to capture the relationship of inputs better than a one-sided RNN architecture. Indeed, sharing the features that are learned by each basic unit reduces the misclassification and improves overall accuracy. Also, considering the same inputs for all basic units enhances the classifier performance by providing a prior information about the inputs. In other words, the uncertainty that exist between the basic units is reduced in BRNN compared to RNN.

Again, various architectures of $\mathrm{RNN}$ at different signal lengths are compared for eight-channel (8C) dataset. BRNN with the same inputs and RNN with the sequential inputs show better performance than other architectures. Both architectures achieve the accuracy of $93.3 \%$ in $500 \mathrm{msec}$. These results are summarized in Table IV.

In Fig. 7 and Fig. 8 , the classification accuracy of $2 \mathrm{C}$ and $8 \mathrm{C}$ datasets for BRNN with the same inputs are shown. For the $2 \mathrm{C}$ dataset at the $600 \mathrm{msec}$ signal length, the accuracy of ring, thumb-index, thumb-middle and thumb-little is $100 \%$. For $8 \mathrm{C}$ dataset at the 500 msec signal length, the accuracy for thumb, index, middle, little, thumb-middle, thumb-ring, middle-ring and index-middle-ring is $100 \%$. Comparing the accuracies of classes, a uniform accuracy for each finger movement in both datasets is observed. Such uniform accuracy for different classes is important factor that proves the proposed BRNN architecture has stable performance on various finger 
TABLE III

THE ACCURACY (IN \%) OF DIFFERENT RNN STRUCTURES FOR VARIOUS SIGNAL LENGTH FOR 2C DATASET.

\begin{tabular}{lccccccccccc}
\hline \multirow{2}{*}{ RNN Structures } & \multicolumn{10}{c}{ Signal length (msec) } \\
\cline { 2 - 13 } & 100 & 150 & 200 & 250 & 300 & 350 & 400 & 450 & 500 & 550 & 600 \\
\hline \hline RNN with the same inputs & 62.0 & 62.0 & 72.5 & 74.0 & 78.0 & 79.5 & 84.5 & 86.0 & 85.5 & 89.0 & 90.0 \\
\hline BRNN with the same inputs & 70.5 & 70.5 & 74.0 & 81.5 & 87.0 & 90.0 & 91.0 & 92.5 & 92.0 & 91.5 & 96.0 \\
\hline RNN with sequential inputs & - & - & - & - & 85.5 & 85.5 & 88.0 & 89.0 & 91.0 & 93.0 & 93.0 \\
\hline BRNN with sequential inputs & - & - & - & - & 85.0 & 85.0 & 91.0 & 91.5 & 93.5 & 93.5 & 93.5 \\
\hline
\end{tabular}

TABLE IV

THE ACCURACY (IN \%) OF DIFFERENT RNN STRUCTURES FOR VARIOUS SIGNAL LENGTH FOR 8C DATASET.

\begin{tabular}{lccccccccccc}
\hline \multirow{2}{*}{ RNN Structures } & \multicolumn{10}{c}{ Signal length (msec) } \\
\cline { 2 - 12 } & 100 & 150 & 200 & 250 & 300 & 350 & 400 & 450 & 500 & 550 & 600 \\
\hline \hline RNN with the same inputs & 82.5 & 82.5 & 81.6 & 84.1 & 87.5 & 84.1 & 86.6 & 87.5 & 90.8 & 91.6 & 91.6 \\
\hline BRNN with the same inputs & 85.0 & 85.0 & 90.0 & 90.8 & 92.5 & 92.5 & 92.5 & 92.5 & 93.3 & 93.3 & 93.3 \\
\hline RNN with sequential inputs & - & - & - & - & 89.1 & 89.1 & 90.8 & 90.8 & 90.8 & 91.6 & 92.5 \\
\hline BRNN with sequential inputs & - & - & - & - & 89.2 & 89.2 & 90.8 & 90.8 & 93.3 & 93.3 & 93.3 \\
\hline
\end{tabular}

TABLE V

COMPARING THE RESULTS OF DIFFERENT APPROACHES FOR THE SAME (2C) EMG DATASET [16].

\begin{tabular}{|c|c|c|c|c|c|}
\hline Methods & Features & Classifier & Classes & Signal length (msec) & Avg. Acc (in \%) \\
\hline$|30|$ & TD+Hjorth+RMS & ANN & 5 & 5000 & 96.7 \\
\hline [31] & $\mathrm{AR}+\mathrm{RMS}$ & $\mathrm{ANN}+\mathrm{NMF}$ & 5 & 5000 & 92 \\
\hline [11] & Reflection Coefficients & SVM & 10 & 5000 & 89 \\
\hline |32] & FNPA & ELM+libSVM+RegTree & 10 & 5000 & 91 \\
\hline [15] & Reflection Coefficients & EVM & 10 & 5000 & 91 \\
\hline [33] & Mixture of Features & Random Forest & 10 & 5000 & 93.75 \\
\hline [14] & Hudgind+RMS & SVM & 10 & 5000 & 96.67 (just thumb finger) \\
\hline [34] & FrFT & KNN & 10 & 5000 & 98.12 \\
\hline |10] & statistics features+FFT & GB & 10 & 5000 & 98.5 \\
\hline 35 & Mixture of Features & SVM & 10 & 5000 & 96.5 \\
\hline [16] & $\mathrm{TD}+\mathrm{AR}+$ Hjorth & $\mathrm{KNN}+\mathrm{SVM}+$ Fusion & 10 & 800 & 90 \\
\hline |15] & Wavelet & Deep Neural Network & 10 & 800 & 95.5 \\
\hline Ours & Wavelet & BRNN & 10 & 600 & 96 \\
\hline
\end{tabular}

movements.

The accuracy of classification depends on several factors including the number of classes, number of sensors, extracted features and utilized classifier. Increasing the number of classes will decrease the accuracy of classifications. Also, the number of sensors used for the experiments can significantly affect the final result. Therefore, we compare our results with other researchers with the same situation on the same dataset. In Table $\mathrm{V}$, the results of $2 \mathrm{C}$ EMG dataset are compared, while in Table VI, the 8C EMG dataset is considered. The offline results have generally better accuracies but as it is discussed, for real world applications and motion control of prosthetics, online classification have practical role. Rare researches have been done on the online classification of EMG signals. Our result is very competitive such that the accuracy and signal length are simultaneously regarded and better accuracy in less signal length is gained for both datasets.

We have trained this model in python and ran it in a windows-based PC with $2.60 \mathrm{GHz}$ CPU and $16 \mathrm{~GB}$ memory. The delay time for the system is the result of feature extraction and process time required by the classifier to make the decision. For 2C dataset using BRNN at $600 \mathrm{msec}$ signal length at postprocessing, delay time is about $4.72 \mathrm{msec}$ for the feature extraction and $3.12 \mathrm{msec}$ for the classification. 
TABLE VI

COMPARING THE RESULTS OF DIFFERENT APPROACHES FOR THE SAME (8C) EMG DATASET [23].

\begin{tabular}{cccccc}
\hline Methods & Features & Classifier & Classes & Signal length (msec) & Avg. Acc (in \%) \\
\hline \hline$[23$ & MCA & SVM+KNN+ELM & 15 & 20000 & 95.0 \\
\hline 36 & Mixture of Features & $\begin{array}{l}\text { NB+KNN+MLP+ } \\
\text { QDA+SVM+ELM }\end{array}$ & 15 & 20000 & 90 \\
\hline 37 & WPT-4+TD+AR-4+RMS & QDA+KNN+SVM & 15 & 20000 & 98.5 \\
\hline 38 & Raw Data & CNN & 15 & 100 & 91.26 \\
\hline Ours & Wavelet & BRNN & $\mathbf{1 5}$ & $\mathbf{5 0 0}$ & $\mathbf{9 3 . 3 3}$ \\
\hline
\end{tabular}

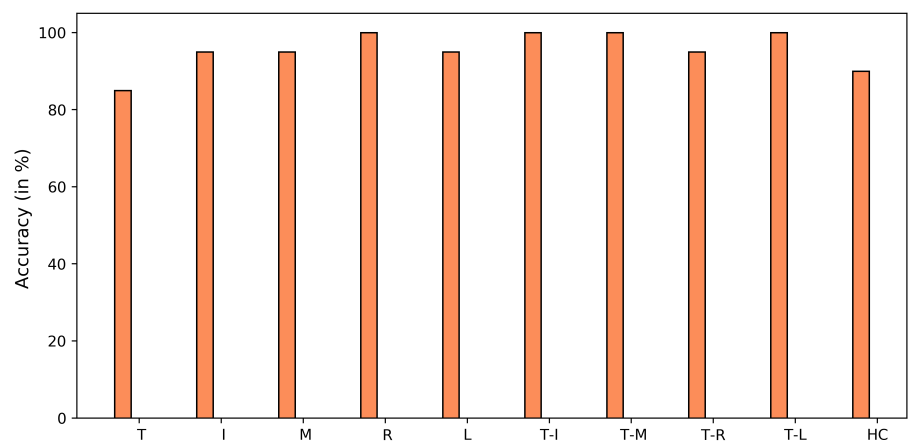

Fig. 7. The classification accuracy for each of 10 classes of BRNN for $2 \mathrm{C}$ dataset at signal length of $600 \mathrm{msec}$.

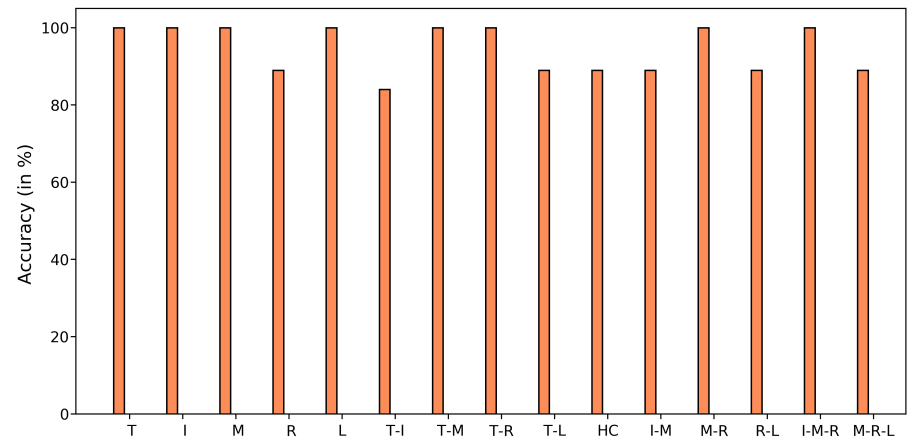

Fig. 8. The classification accuracy for each of 15 classes of BRNN for $8 \mathrm{C}$ dataset at signal length of 500 msec.

\section{CONCLUSION}

To overcome the challenges of real-time classification of EMG signals, we introduced two new recurrent neural network-based architectures. Discrete wavelet transformation was utilized to extract the features from time-frequency domain to feed into the recurrent neural networks. Each architecture was investigated for two different input forms. The performance of each architecture was evaluated and it was compared with the state-of-the-art approaches. It was shown that at least one of these architectures increases the classification accuracy, reduces the delay time, and outperforms other architectures.

\section{REFERENCES}

[1] S. Rouhani, T. Rahman, and V. Gogate, "Algorithms for the nearest assignment problem.," in IJCAI, pp. 5096-5102, 2018.

[2] M. Esmaeili, N. Granpayeh, and M. Bozorgi, "A novel reliable optimization method for output beam forming of photonic crystal waveguide terminated with surface crow," Optik, vol. 126, no. 4, pp. 421-425, 2015.

[3] A. A. M. Lima, R. M. Araujo, F. A. G. d. Santos, V. H. Yoshizumi, F. K. H. de Barros, D. H. Spatti, L. H. B. Liboni, and M. E. Dajer, "Classification of hand movements from emg signals using optimized mlp," in 2018 International Joint Conference on Neural Networks (IJCNN), pp. 1-7, 2018. 
[4] D. L. Espinoza and L. Elí Sánchez Velasco, "Comparison of emg signal classification algorithms for the control of an upper limb prosthesis prototype," in 2020 17th International Conference on Electrical Engineering, Computing Science and Automatic Control (CCE), pp. 1-4, 2020.

[5] Z. Li, B. Wang, C. Yang, Q. Xie, and C.-Y. Su, "Boosting-based emg patterns classification scheme for robustness enhancement," IEEE Journal of Biomedical and Health Informatics, vol. 17, no. 3, pp. 545$552,2013$.

[6] K. Nazarpour, A. R. Sharafat, and S. M. P. Firoozabadi, "Application of higher order statistics to surface electromyogram signal classification," IEEE Transactions on Biomedical Engineering, vol. 54, no. 10, pp. 1762-1769, 2007.

[7] Z. Taghizadeh, S. Rashidi, and A. Shalbaf, "Finger movements classification based on fractional fourier transform coefficients extracted from surface emg signals," Biomedical Signal Processing and Control, vol. 68, pp. 102573-102580, 2021.

[8] L. S. Praveen, S. N. Nagananda, and P. Shankapal, "Design and development of real time bionic hand control using emg signal," in 2018 IEEE International Conference on Electronics, Computing and Communication Technologies (CONECCT), pp. 1-4, 2018.

[9] A. G. Jaramillo and M. E. Benalcázar, "Real-time hand gesture recognition with emg using machine learning," in 2017 IEEE Second Ecuador Technical Chapters Meeting (ETCM), pp. 1-5, IEEE, 2017.

[10] C. K. Bhattachargee, N. Sikder, M. T. Hasan, and A.-A. Nahid, "Finger movement classification based on statistical and frequency features extracted from surface emg signals," in 2019 International Conference on Computer, Communication, Chemical, Materials and Electronic Engineering (IC4ME2), pp. 1-4, IEEE, 2019.

[11] M. Heydarzadeh, J. Birjandtalab, and M. Nourani, "Emg spectral analysis for prosthetic finger control," in 2017 European Conference on Electrical Engineering and Computer Science (EECS), pp. 131-135, 2017.

[12] A. Gailey, P. Artemiadis, and M. Santello, "Proof of concept of an online emg-based decoding of hand postures and individual digit forces for prosthetic hand control," Frontiers in neurology, vol. 8, pp. 7-21, 2017.

[13] R. B. Azhiri, M. Esmaeili, and M. Nourani, "Emg-based feature extraction and classification for prosthetic hand control," arXiv preprint arXiv: 2107.00733, 2021.

[14] N. M. Esa, A. M. Zain, and M. Bahari, "Electromyography (emg) based classification of finger movements using svm," International Journal of Innovative Computing, vol. 8, no. 3, 2018.

[15] R. B. Azhiri, M. Esmaeili, M. Jafarzadeh, and M. Nourani, "Emg signal classification using reflection coefficients and extreme value machine," arXiv preprint arXiv: 2106.10561, 2021.

[16] R. N. Khushaba, S. Kodagoda, M. Takruri, and G. Dissanayake, "Toward improved control of prosthetic fingers using surface electromyogram (emg) signals," Expert Systems with Applications, vol. 39, no. 12 pp. 10731-10738, 2012.

[17] J.-U. Chu, I. Moon, and M.-S. Mun, "A real-time emg pattern recognition system based on linear-nonlinear feature projection for a multifunction myoelectric hand," IEEE Transactions on biomedical engineering, vol. 53, no. 11, pp. 2232-2239, 2006.

[18] D. Xiong, D. Zhang, X. Zhao, and Y. Zhao, "Deep learning for emgbased human-machine interaction: A review," IEEE/CAA Journal of Automatica Sinica, vol. 8, no. 3, pp. 512-533, 2021.

[19] N. Nasri, S. Orts-Escolano, F. Gomez-Donoso, and M. Cazorla, "Inferring static hand poses from a low-cost non-intrusive semg sensor," Sensors, vol. 19, no. 2, p. 371, 2019.

[20] P. Koch, H. Phan, M. Maass, F. Katzberg, R. Mazur, and A. Mertins, "Recurrent neural networks with weighting loss for early prediction of hand movements," in 2018 26th European Signal Processing Conference (EUSIPCO), pp. 1152-1156, IEEE, 2018.

[21] M. Simão, P. Neto, and O. Gibaru, "Emg-based online classification of gestures with recurrent neural networks," Pattern Recognition Letters, vol. 128, pp. 45-51, 2019.

[22] M. Esmaeili and A. Nosratinia, "Semi-supervised node classification by graph convolutional networks and extracted side information," arXiv preprint arXiv:2009.13734, 2020.

[23] R. N. Khushaba and S. Kodagoda, "Electromyogram (emg) feature reduction using mutual components analysis for multifunction prosthetic fingers control," in 2012 12th International Conference on Control Automation Robotics \& Vision (ICARCV), pp. 1534-1539, IEEE, 2012.
[24] S. Rouhani, T. Rahman, and V. Gogate, "A novel approach for constrained optimization in graphical models," Advances in Neural Information Processing Systems, vol. 33, 2020.

[25] M. Zardoshti-Kermani, B. C. Wheeler, K. Badie, and R. M. Hashemi, "Emg feature evaluation for movement control of upper extremity prostheses," IEEE Transactions on Rehabilitation Engineering, vol. 3, no. 4, pp. 324-333, 1995

[26] B. Hudgins, P. Parker, and R. N. Scott, "A new strategy for multifunction myoelectric control," IEEE transactions on biomedical engineering, vol. 40, no. 1, pp. 82-94, 1993.

[27] A. Phinyomark, P. Phukpattaranont, and C. Limsakul, "Feature reduction and selection for emg signal classification," Expert systems with applications, vol. 39, no. 8, pp. 7420-7431, 2012.

[28] A. Phinyomark, F. Quaine, S. Charbonnier, C. Serviere, F. TarpinBernard, and Y. Laurillau, "Feature extraction of the first difference of emg time series for emg pattern recognition," Computer methods and programs in biomedicine, vol. 117, no. 2, pp. 247-256, 2014.

[29] H. R. Al Ghayab, Y. Li, S. Siuly, and S. Abdulla, "A feature extraction technique based on tunable q-factor wavelet transform for brain signal classification," Journal of neuroscience methods, vol. 312, pp. 43-52, 2019.

[30] M. Ariyanto, W. Caesarendra, K. A. Mustaqim, M. Irfan, J. A. Pakpahan, J. D. Setiawan, and A. R. Winoto, "Finger movement pattern recognition method using artificial neural network based on electromyography (emg) sensor," in 2015 International Conference on Automation, Cognitive Science, Optics, Micro Electro-Mechanical System, and Information Technology (ICACOMIT), pp. 12-17, IEEE, 2015.

[31] G. R. Naik and H. T. Nguyen, "Nonnegative matrix factorization for the identification of emg finger movements: Evaluation using matrix analysis," IEEE journal of biomedical and health informatics, vol. 19, no. 2, pp. 478-485, 2014.

[32] R. N. Khushaba, S. Kodagoda, D. Liu, and G. Dissanayake, "Electromyogram (emg) based fingers movement recognition using neighborhood preserving analysis with qr-decomposition," in 2011 Seventh International Conference on Intelligent Sensors, Sensor Networks and Information Processing, pp. 1-105, IEEE, 2011.

[33] W. Al-Sharu and A. M. Alqudah, "Enhancing prediction of prosthetic fingers movement based on semg using mixtures of features and random forest," International Journal of Recent Technology and Engineering, vol. 8, no. 4, pp. 289-294, 2019.

[34] Z. Taghizadeh, S. Rashidi, and A. Shalbaf, "Finger movements classification based on fractional fourier transform coefficients extracted from surface emg signals," Biomedical Signal Processing and Control, vol. 68, p. 102573, 2021.

[35] N. Phukan, N. M. Kakoty, P. Shivam, and J. Q. Gan, "Finger movements recognition using minimally redundant features of wavelet denoised emg," Health and Technology, vol. 9, no. 4, pp. 579-593, 2019.

[36] S. Shin, R. Langari, and R. Tafreshi, "A performance comparison of emg classification methods for hand and finger motion," in Dynamic Systems and Control Conference, vol. 46193, p. V002T16A008, American Society of Mechanical Engineers, 2014.

[37] S. Bhagwat and P. Mukherji, "Electromyogram (emg) based fingers movement recognition using sparse filtering of wavelet packet coefficients," Sādhanāa, vol. 45 , no. 1 , pp. 1-11, 2020.

[38] M. Jafarzadeh, D. C. Hussey, and Y. Tadesse, "Deep learning approach to control of prosthetic hands with electromyography signals," in 2019 IEEE International Symposium on Measurement and Control in Robotics (ISMCR), pp. A1-4, IEEE, 2019. 